\title{
Gaps in Sexually Transmitted Infection Screening Among Men Who Have Sex With Men in Pre-exposure Prophylaxis (PrEP) Care in the United States
}

\author{
Christina Chandra, ${ }^{1}$ Kevin M. Weiss, ${ }^{1}$ Colleen F. Kelley, ${ }^{2,}$ Julia L. Marcus, ${ }^{3}$ and Samuel M. Jenness ${ }^{1, \oplus}$ \\ ${ }^{1}$ Department of Epidemiology, Rollins School of Public Health, Emory University, Atlanta, Georgia, USA, ${ }^{2}$ Department of Medicine, Division of Infectious Diseases, Emory University School of
} Medicine, Atlanta, Georgia, USA, and ${ }^{3}$ Harvard Medical School and Harvard Pilgrim Health Care Institute, Boston, Massachusetts, USA

Background. The US Centers for Disease Control and Prevention (CDC) recommends comprehensive sexually transmitted infection (STI) screening every 3-6 months for men who have sex with men (MSM) using human immunodeficiency virus (HIV) pre-exposure prophylaxis (PrEP). The gaps between these recommendations and clinical practice by region have not been quantified.

Methods. We used survey data collected from the internet-based ARTnet study between 2017 and 2019 on STI screening among MSM across the United States, stratified by current, prior, and never PrEP use. Poisson regression models with robust error variance were used to model factors, including residence in the Southeast, associated with consistent ("always" or "sometimes") exposure sitespecific STI screening during PrEP care.

Results. Of 3259 HIV-negative MSM, 19\% were currently using PrEP, 6\% had used PrEP in the past, and 75\% had never used PrEP. Among ever PrEP users, 87\%, 78\%, 57\%, and 64\% reported consistent screening for STIs by blood sample, urine sample or urethral swab, rectal swab, or pharyngeal swab, respectively, during PrEP care. Compared to PrEP users in all other regions, PrEP users in the Southeast were significantly less likely to be consistently screened for urogenital (adjusted prevalence ratio [aPR], 0.86; 95\% confidence interval [CI], .76-.98) and rectal STIs (aPR, 0.76; 95\% CI, .62-.93) during PrEP care.

Conclusions. Substantial gaps exist between CDC recommendations for STI screening during PrEP care and current clinical practice, particularly for rectal and pharyngeal exposure sites that can harbor asymptomatic infections and for MSM in Southeast states where the STI burden is substantial.

Keywords. HIV; pre-exposure prophylaxis; sexually transmitted infections; screening; men who have sex with men.

In the United States, cases of the major reportable bacterial sexually transmitted infections (STIs)-syphilis, gonorrhea, and chlamydia-increased by up to $71 \%$ between 2014 and 2018 , reaching all-time highs [1]. Rates of new human immunodeficiency virus (HIV) diagnoses have remained stable overall but increased in key subgroups during this time [2]. Men who have sex with men (MSM) are disproportionately affected by STIs and HIV, accounting for more than half of new infectious syphilis cases, $40 \%$ of gonorrhea cases, and $66 \%$ of HIV cases in 2018 despite only representing $4 \%$ of the population [1-3]. Additionally, more than half of new HIV diagnoses in 2018 occurred in the South, which also has the highest STI rates $[1,2]$.

One hypothesis for the increase in STI cases among MSM has been the growing use of HIV pre-exposure prophylaxis (PrEP). In a meta-analysis, the pooled STI prevalence among

Received 11 March 2020; editorial decision 24 June 2020; accepted 17 July 2020; published online July 23, 2020.

Correspondence: S. M. Jenness, Emory University, 1520 Clifton Rd, Atlanta, GA 30323 (samuel.m.jenness@emory.edu).

Clinical Infectious Diseases $^{\circledR} \quad$ 2021;73(7):e2261-9

(C) The Author(s) 2020. Published by Oxford University Press for the Infectious Diseases Society of America. All rights reserved. For permissions, e-mail: journals.permissions@oup.com. DOI: 10.1093/cid/ciaa1033
PrEP users within 3 months of PrEP initiation was 24\%, and STI incidence was estimated at 72 per 100 person-years among persistent PrEP users globally [4]. US studies have found that STI prevalence or incidence increased among MSM after PrEP initiation $[5,6]$. The difference in STI rates before and after PrEP initiation could result from multiple factors, including increased detection of cases from more frequent screening in PrEP care, changes in sexual risk behavior after PrEP initiation ("risk compensation"), and trends in sexual behavior and STI rates that predate PrEP [1, 7-10]. Many PrEP users are at elevated risk of STIs because PrEP is recommended for MSM with a history of inconsistent condom use and multiple partners [11].

The US Centers for Disease Control and Prevention (CDC) recommends comprehensive STI screening every 3-6 months for MSM using PrEP [11]. This includes blood tests to screen for syphilis, and urine samples and urethral, pharyngeal, and rectal swabs for gonorrhea and chlamydia infections. Prior modeling research suggested that although STI diagnoses may initially increase with expanded PrEP use due to variations of risk compensation, routine STI screening and treatment during PrEP care could prevent over $40 \%$ of gonorrhea and chlamydia cases among MSM over 10 years [12]. These results were partially attributed to increased treatment 
of asymptomatic STIs that would have otherwise been undiagnosed. These projections assumed complete adherence to PrEP-related STI screening and treatment recommendations; secondary model scenarios projected that STI incidence could increase if fewer than 50\% of PrEP users were consistently screened and treated for STIs.

Quantifying any gaps between PrEP STI screening recommendations and clinical practice is therefore critical to understanding the role of PrEP care in STI control. Several studies have evaluated this in different regions, finding lower rates of STI screening than recommended [7, 13-16]. Although these studies provide important evidence regarding gaps in PrEP care, there are still no national-level studies of MSM that have compared these gaps across geographic regions. Geographic heterogeneity in clinical practice is important to understand because of the regional differences in infrastructure for PrEP services (eg, the ability to assess kidney function and serological confirmation of hepatitis B status for PrEP eligibility) and the spatial clustering of STIs in the Southeast [17, 18]. Differences in STI screening practices may also reflect state-level factors that impact PrEP care, including access to healthcare (eg, Medicaid expansion) and social determinants of health that may vary by state (eg, housing stability, employment) $[19,20]$. By examining these differences, we may better understand the role that geographically focused efforts-such as the federal Ending the HIV Epidemic initiative-may play in stemming both the HIV and STI epidemics in the Southeast [21].

In this study, we compared STI screening among MSM by PrEP use history in a study of MSM in the United States. We then evaluated factors associated with consistent STI screening at PrEP care visits, including residence in the Southeast and recent sexual exposure at anatomical sites. We hypothesized that there would be strong geographic differences in STI screening consistency among PrEP users even after controlling for demographic and behavioral factors.

\section{METHODS}

\section{Study Design}

ARTnet was a cross-sectional web-based study of MSM in the United States conducted between 2017 and 2019 [22]. The study recruited participants through the national American Men's Internet Survey (AMIS), with a response rate of 53\% (4904/9295) [23]. ARTnet eligibility criteria included male sex at birth, current male identity, lifetime history of sexual activity with another man, and age between 15 and 65 years. The study, provided only in English, surveyed participants about recent sexual behaviors, HIV and STI screening, and egocentric network data [24]. We restricted the participants in this analysis to PrEP-eligible MSM, defined as MSM who had ever had an HIV test and who self-reported as HIV-negative. The Emory
University Institutional Review Board reviewed and approved the study.

\section{Measures}

We quantified participant-reported data on HIV and STI screening from blood samples, urine sample/urethral swabs, and rectal and pharyngeal swabs at PrEP care visits and any HIV or STI screening in the past 12 months. Participants who had ever used PrEP were asked: "At these PrEP (ie, Truvada) check-up visits, how often did you get tested for HIV and sexually transmitted diseases (STDs)? STDs could include gonorrhea, chlamydia, and syphilis." Participants could answer "Always," "Sometimes," "Rarely," or "Never" for HIV screening or STI screening site/method: throat (pharyngeal swab), anus (rectal swab), genital/penis (urine sample/urethral swab), or blood sample. We assumed blood-based STI screening was used to possibly screen for syphilis, because blood samples are necessary for syphilis screening whereas swabs and urine samples are used for nucleic acid amplification tests (NAATs), the gold standard for gonorrhea and chlamydia testing. For statistical models, we dichotomized the outcomes to consistent screening ("Always" or "Sometimes") versus inconsistent screening ("Rarely" or "Never").

We then investigated correlates of consistent STI screening. We considered age group, race/ethnicity, current health insurance, annual household income, recent sexual exposure at anatomical site, and geographic region of residence. Using data on the 5 most recent reported partners in the past year, respondents were classified as having been exposed at the urethra (any insertive anal or insertive oral intercourse), rectum (any receptive anal intercourse), or pharynx (any receptive oral intercourse). For geography, we considered census division and state by matching reported residential ZIP codes against census data [22]. Our primary exposure of interest was residence in 12 Southeast states as defined by the US Bureau of Economic Analysis: Alabama, Arkansas, Florida, Georgia, Kentucky, Louisiana, Mississippi, North Carolina, South Carolina, Tennessee, Virginia, and West Virginia [25].

\section{Statistical Analysis}

Our primary research objective was to quantify the association between residence in Southeast states and consistent screening for STIs among MSM who were currently using or had previously used PrEP. We first estimated the bivariable prevalence ratios between our hypothesized correlates-age, race/ethnicity, current health insurance, annual household income, recent sexual exposure at anatomical site, and geography_and consistent site-specific STI screening using Poisson regression with robust error variance and complete case analysis. In multivariable Poisson regression models, we estimated the prevalence ratios between our primary exposure (residence in a Southeast state) and consistent STI screening adjusted for 4 variables that we 
hypothesized would confound this relationship: age, race/ethnicity, annual household income, and sexual exposure at anatomical site. We did not control for variables that we considered mediators of the associations, such as health insurance that may vary by state as a result of Medicaid expansion. All analyses were conducted in R 3.6.1 [26]. Analysis scripts are provided in a GitHub repository (https://github.com/EpiModel/PrEP-STITest), and the primary data are available upon request.

\section{RESULTS}

After excluding PrEP-ineligible participants, 3259 MSM were included in this analysis. Of those, $631(19 \%)$ were currently using PrEP at the time of survey completion, 178 (6\%) had previously used PrEP, and 2450 (75\%) had never used PrEP. Table 1 presents the demographics and HIV/STI screening history by PrEP use. Overall, 74\% were non-Hispanic white, 51\% were aged 15-34 years, and 23\% resided in Southeast states.

\section{Table 1. Characteristics of Pre-exposure Prophylaxis (PrEP)-Eligible ARTnet Participants, Stratified by PrEP Use History}

\begin{tabular}{|c|c|c|c|c|c|c|c|c|}
\hline & \multicolumn{2}{|c|}{ Total $^{a}$} & \multicolumn{2}{|c|}{ Current PrEP Use } & \multicolumn{2}{|c|}{ Prior PrEP Use } & \multicolumn{2}{|c|}{ Never Used PrEP } \\
\hline & N or Mean & $\%^{b}$ or SD & $N$ or Mean & $\%$ or SD & $\mathrm{N}$ or Mean & $\%$ or SD & $\mathrm{N}$ or Mean & $\%$ or SD \\
\hline Total sample & 3259 & 100.0 & 631 & 100.0 & 178 & 100.0 & 2450 & 100.0 \\
\hline \multicolumn{9}{|l|}{ Race/ethnicity } \\
\hline Black (non-Hispanic) & 134 & 4.1 & 28 & 4.4 & 4 & 2.3 & 102 & 4.2 \\
\hline Hispanic & 422 & 12.9 & 81 & 12.8 & 23 & 12.9 & 318 & 13.0 \\
\hline Other (non-Hispanic) & 283 & 8.7 & 53 & 8.4 & 11 & 6.2 & 219 & 8.9 \\
\hline White (non-Hispanic) & 2420 & 74.3 & 469 & 74.3 & 140 & 78.7 & 1811 & 73.9 \\
\hline Age & 37.3 & 13.6 & 39.5 & 12.4 & 34.1 & 11.5 & 36.9 & 13.9 \\
\hline $15-24$ & 713 & 21.9 & 71 & 11.3 & 36 & 20.2 & 606 & 24.7 \\
\hline $25-34$ & 947 & 29.1 & 196 & 31.1 & 76 & 42.7 & 675 & 27.6 \\
\hline $35-44$ & 518 & 15.9 & 122 & 19.3 & 26 & 14.6 & 370 & 16.3 \\
\hline $45-54$ & 573 & 17.6 & 149 & 23.6 & 24 & 13.5 & 400 & 16.3 \\
\hline $55-65$ & 508 & 15.6 & 93 & 14.7 & 16 & 9.0 & 399 & 16.3 \\
\hline \multicolumn{9}{|c|}{ Residence in census division ${ }^{c}$} \\
\hline Pacific & 562 & 17.2 & 155 & 24.6 & 31 & 17.4 & 376 & 15.3 \\
\hline Mountain & 281 & 8.6 & 30 & 4.8 & 21 & 11.8 & 230 & 9.4 \\
\hline West North Central & 187 & 5.7 & 32 & 5.1 & 13 & 7.3 & 142 & 5.8 \\
\hline East North Central & 466 & 14.3 & 92 & 14.6 & 25 & 14.0 & 349 & 14.2 \\
\hline West South Central & 323 & 9.9 & 61 & 9.7 & 11 & 6.2 & 251 & 10.2 \\
\hline East South Central & 132 & 4.1 & 19 & 3.0 & 6 & 3.4 & 107 & 4.4 \\
\hline South Atlantic & 701 & 21.5 & 119 & 18.9 & 35 & 19.7 & 547 & 22.3 \\
\hline Middle Atlantic & 442 & 13.6 & 92 & 14.6 & 20 & 11.2 & 330 & 13.5 \\
\hline New England & 165 & 5.1 & 31 & 4.9 & 16 & 9.0 & 118 & 4.8 \\
\hline \multicolumn{9}{|c|}{ Residence in Southeast ${ }^{d}$} \\
\hline Yes & 760 & 23.3 & 121 & 19.2 & 33 & 18.5 & 606 & 24.7 \\
\hline No & 2499 & 76.7 & 510 & 80.8 & 145 & 81.5 & 1844 & 75.3 \\
\hline \multicolumn{9}{|l|}{ Health insurance } \\
\hline Private & 2441 & 76.4 & 516 & 82.2 & 128 & 71.9 & 1797 & 75.2 \\
\hline Public & 514 & 16.1 & 94 & 15.0 & 33 & 18.5 & 387 & 16.2 \\
\hline None & 242 & 7.6 & 18 & 2.9 & 17 & 9.6 & 207 & 8.7 \\
\hline \multicolumn{9}{|c|}{ Annual household income } \\
\hline$\$ 0$ to $\$ 19999$ & 372 & 12.3 & 54 & 9.3 & 18 & 10.7 & 300 & 13.2 \\
\hline$\$ 20000$ to $\$ 39999$ & 574 & 19.1 & 93 & 16.1 & 34 & 20.2 & 447 & 19.7 \\
\hline$\$ 40000$ to $\$ 74999$ & 861 & 28.6 & 153 & 26.4 & 56 & 33.3 & 652 & 28.8 \\
\hline$\$ 75000$ or more & 1206 & 40.0 & 279 & 48.2 & 60 & 35.7 & 867 & 38.3 \\
\hline \multicolumn{9}{|c|}{ Screened for HIV in past 12 months } \\
\hline Yes & 2106 & 71.9 & 561 & 98.2 & 129 & 79.6 & 1416 & 64.5 \\
\hline No & 822 & 28.1 & 10 & 1.8 & 33 & 20.4 & 779 & 35.5 \\
\hline \multicolumn{9}{|c|}{ Screened for an STI in past 12 months } \\
\hline Yes & 1677 & 54.4 & 547 & 89.1 & 122 & 70.9 & 1008 & 43.8 \\
\hline No & 1408 & 45.6 & 67 & 10.9 & 50 & 28.1 & 1291 & 56.2 \\
\hline
\end{tabular}

Abbreviations: HIV, human immunodeficiency virus; PrEP, pre-exposure prophylaxis; SD, standard deviation; STI, sexually transmitted infection; USD, US dollars.

${ }^{a}$ Totals may not add up to sample total due to missing data for health insurance, annual household income, and screening for HIV and STIs in the past 12 months.

${ }^{b}$ Column percents.

${ }^{c}$ https://www2.census.gov/geo/pdfs/maps-data/maps/reference/us_regdiv.pdf.

d Residence in Alabama, Arkansas, Florida, Georgia, Kentucky, Louisiana, Mississippi, North Carolina, South Carolina, Tennessee, Virginia, and West Virginia. 
Residents in the Southeast were generally underrepresented, accounting for 25\% of MSM who had never used PrEP and $19 \%$ of both current and prior PrEP users. Four-fifths (82\%) of current PrEP users reported having any private health insurance compared with $72 \%$ of prior PrEP users and $75 \%$ of never PrEP users. Only 3\% of current PrEP users were uninsured compared with $10 \%$ of prior PrEP users and 9\% of never PrEP users. A higher proportion of current PrEP users reported screening for a bacterial STI (without specifying specimen collected) in the past year (89\%), compared to prior PrEP users (71\%) and never PrEP users (44\%). Similarly, 98\% of current PrEP users were screened for HIV in the past year compared to $80 \%$ of prior PrEP users and 65\% of never PrEP users.
Among MSM who had ever used PrEP, 84\% of current users reported attending PrEP care visits at least every 3 months compared to $71 \%$ of prior PrEP users (Table 2). Overall, $91 \%$ of MSM who had ever used PrEP were always screened for HIV at their PrEP care visits. Greater proportions of current PrEP users, compared to prior PrEP users, reported "always" being screened for STIs by throat swab (44\% vs $35 \%$ ), rectal swab ( $37 \%$ vs $32 \%$ ), blood (possibly for syphilis; $70 \%$ vs $57 \%$ ), and urine sample/urethral swab (59\% vs 50\%) at PrEP care visits. In contrast, MSM who had ever used PrEP had also reported "never" providing a rectal swab (35\%), throat swab (28\%), and urine sample/urethral swab (17\%) for STI screening during PrEP care visits.

Table 2. Frequency of Pre-exposure Prophylaxis (PrEP) Care Visits and HIV/STI Screening at PrEP Care Visits Among Men Who Have Sex with Men With Current or Prior PrEP Use

\begin{tabular}{|c|c|c|c|c|c|c|}
\hline & \multicolumn{2}{|c|}{ Total $^{a}$} & \multicolumn{2}{|c|}{ Current PrEP Use } & \multicolumn{2}{|c|}{ Prior PrEP Use } \\
\hline & N & $\%$ & N & $\%$ & N & $\%$ \\
\hline Total sample & 809 & 100.0 & 631 & 100.0 & 178 & 100.0 \\
\hline \multicolumn{7}{|l|}{ Frequency of PrEP care visits } \\
\hline Every month & 23 & 2.9 & 13 & 2.1 & 10 & 5.7 \\
\hline Every 3 months & 629 & 78.3 & 514 & 82.0 & 115 & 65.3 \\
\hline Every 6 months & 83 & 10.3 & 75 & 12.0 & 8 & 4.5 \\
\hline Every 9 months & 3 & 0.4 & 1 & 0.2 & 2 & 1.1 \\
\hline Every 12 months & 8 & 1.0 & 8 & 1.3 & 0 & 0.0 \\
\hline Returned only once & 21 & 2.6 & 3 & 0.5 & 18 & 10.2 \\
\hline Returned at some other interval of time & 11 & 1.4 & 6 & 1.0 & 5 & 2.8 \\
\hline Did not return regularly & 25 & 3.1 & 7 & 1.1 & 18 & 10.2 \\
\hline Frequency of HIV screening at PrEP care visits & & 0 & & & & \\
\hline Always & 685 & 91.1 & 566 & 92.5 & 119 & 85.0 \\
\hline Sometimes & 50 & 6.6 & 40 & 6.5 & 10 & 7.1 \\
\hline Rarely & 7 & 0.9 & 3 & 0.5 & 4 & 2.9 \\
\hline Never & 10 & 1.3 & 3 & 0.5 & 7 & 5.0 \\
\hline \multicolumn{7}{|c|}{ Frequency of throat swab for STI screening at PrEP care visits } \\
\hline Always & 316 & 42.5 & 267 & 44.1 & 49 & 35.3 \\
\hline Sometimes & 161 & 21.6 & 134 & 22.1 & 27 & 19.4 \\
\hline Rarely & 56 & 7.5 & 44 & 7.3 & 12 & 8.6 \\
\hline Never & 211 & 28.4 & 160 & 26.4 & 51 & 36.7 \\
\hline \multicolumn{7}{|c|}{ Frequency of rectal swab for STI screening at PrEP care visits } \\
\hline Always & 270 & 36.2 & 226 & 37.2 & 44 & 31.7 \\
\hline Sometimes & 155 & 20.8 & 128 & 21.1 & 27 & 19.4 \\
\hline Rarely & 58 & 7.8 & 50 & 8.2 & 8 & 5.8 \\
\hline Never & 263 & 35.3 & 203 & 33.4 & 60 & 43.2 \\
\hline \multicolumn{7}{|c|}{ Frequency of blood sample for STI screening at PrEP care visits } \\
\hline Always & 492 & 67.4 & 415 & 69.9 & 77 & 56.6 \\
\hline Sometimes & 143 & 19.6 & 114 & 19.2 & 29 & 21.3 \\
\hline Rarely & 32 & 4.4 & 21 & 3.5 & 11 & 8.1 \\
\hline Never & 63 & 8.6 & 44 & 7.4 & 19 & 14.0 \\
\hline \multicolumn{7}{|c|}{ Frequency of urine sample/urethral swab for STI screening at PrEP care visits } \\
\hline Always & 425 & 57.3 & 356 & 59.0 & 69 & 49.6 \\
\hline Sometimes & 150 & 20.2 & 128 & 21.2 & 22 & 15.8 \\
\hline Rarely & 42 & 5.7 & 33 & 5.5 & 9 & 6.5 \\
\hline Never & 125 & 16.8 & 86 & 14.3 & 39 & 28.1 \\
\hline
\end{tabular}

Abbreviations: HIV, human immunodeficiency virus; PrEP, pre-exposure prophylaxis; STI, sexually transmitted infection.

${ }^{a}$ Totals may not add up to sample total due to missing data. 
A higher percentage of MSM who had ever used PrEP reported consistent possible screening for syphilis (87\%) and urogenital STI screening (78\%) (Table 3) compared to pharyngeal (64\%) and rectal STI screening (57\%) (Table 4). Compared to MSM aged 15-24 years, older MSM aged 55-65 years had the lowest levels of consistent screening for urogenital STIs ( $80 \%$ vs $71 \%$ ), pharyngeal STIs ( $70 \%$ vs $60 \%$ ), and rectal STIs (67\% vs
53\%) (Tables 3 and 4). Consistent screening was reported less in Southeast states for urogenital STI screening ( $66 \%$ vs $80 \%$ in all other states), pharyngeal STI screening (55\% vs $66 \%)$, and rectal STI screening ( $44 \%$ vs $60 \%$ ). Respondents reporting recent exposure at corresponding anatomical sites also reported more consistent screening for urogenital ( $78 \%$ vs $68 \%$ among those unexposed) and rectal STIs ( $60 \%$ vs $44 \%$ ). Proportions of

Table 3. Bivariable Associations of Consistent Possible Syphilis and Urogenital Sexually Transmitted Infection Screening With Demographic and Behavioral Factors Among Men Who Have Sex With Men Who Have Ever Used Pre-exposure Prophylaxis

\begin{tabular}{|c|c|c|c|c|c|c|c|c|c|c|c|c|}
\hline & \multicolumn{6}{|c|}{ Possible Syphilis Screening $^{\mathrm{a}}(\mathrm{N}=730)$} & \multicolumn{6}{|c|}{ Urogenital STI Screening $(N=742)$} \\
\hline & \multicolumn{2}{|c|}{ Consistent $^{\mathrm{b}}$} & \multicolumn{2}{|c|}{ Inconsistent } & \multirow[b]{2}{*}{ PR } & \multirow[b]{2}{*}{$95 \% \mathrm{Cl}$} & \multicolumn{2}{|c|}{ Consistent } & \multicolumn{2}{|c|}{ Inconsistent } & \multirow[b]{2}{*}{ PR } & \multirow[b]{2}{*}{$95 \% \mathrm{Cl}$} \\
\hline & $\mathrm{N}$ & $\%$ & N & $\%$ & & & $\mathrm{~N}$ & $\%$ & $\mathrm{~N}$ & $\%$ & & \\
\hline Total sample & 635 & 87.0 & 95 & 13.0 & & & 575 & 77.5 & 167 & 22.5 & & \\
\hline \multicolumn{13}{|l|}{ Age } \\
\hline $15-24$ & 75 & 83.3 & 15 & 16.7 & 1.00 & & 73 & 80.2 & 18 & 19.8 & 1.00 & $\ldots$ \\
\hline $25-34$ & 215 & 87.8 & 30 & 12.2 & 1.05 & $.95,1.17$ & 201 & 80.1 & 50 & 19.9 & 1.00 & $.89,1.12$ \\
\hline $35-44$ & 123 & 90.4 & 13 & 9.6 & 1.09 & $.97,1.21$ & 104 & 75.4 & 34 & 24.6 & .94 & $.82,1.08$ \\
\hline $45-54$ & 140 & 85.9 & 23 & 14.1 & 1.03 & $.92,1.15$ & 127 & 77.9 & 36 & 22.1 & .97 & $.85,1.11$ \\
\hline $55-65$ & 82 & 85.4 & 14 & 14.6 & 1.02 & $.91,1.16$ & 70 & 70.7 & 29 & 29.3 & .88 & $.75,1.04$ \\
\hline \multicolumn{13}{|l|}{ Race/ethnicity } \\
\hline Black (Non-Hispanic) & 30 & 93.8 & 2 & 6.3 & 1.00 & & 28 & 87.5 & 4 & 12.5 & 1.00 & $\cdots$ \\
\hline Hispanic & 84 & 91.3 & 8 & 8.7 & .97 & $.87,1.09$ & 78 & 84.8 & 14 & 15.2 & .97 & $.83,1.13$ \\
\hline Other (Non-Hispanic) & 53 & 91.4 & 5 & 8.6 & .97 & $.87,1.10$ & 45 & 77.6 & 13 & 22.4 & .89 & $.73,1.07$ \\
\hline White (Non-Hispanic) & 468 & 85.4 & 80 & 14.6 & .91 & $.83,1.00$ & 424 & 75.7 & 136 & 24.3 & 87 & $.75,0.99$ \\
\hline \multicolumn{13}{|l|}{ Health Insurance } \\
\hline None & 28 & 84.8 & 5 & 15.2 & 1.00 & & 23 & 69.7 & 10 & 30.3 & 1.00 & $\ldots$ \\
\hline Public & 98 & 87.5 & 14 & 12.5 & 1.03 & $.88,1.21$ & 91 & 79.8 & 23 & 20.2 & 1.15 & $.90,1.46$ \\
\hline Private & 507 & 87.1 & 75 & 12.9 & 1.03 & $.89,1.19$ & 459 & 77.5 & 133 & 22.5 & 1.11 & $.88,1.40$ \\
\hline \multicolumn{13}{|c|}{ Annual Household Income } \\
\hline$\$ 0$ to $\$ 19999$ & 54 & 83.1 & 11 & 16.9 & 1.00 & & 50 & 76.9 & 15 & 23.1 & 1.00 & \\
\hline$\$ 20000$ to $\$ 39999$ & 86 & 80.4 & 21 & 19.6 & .97 & $.84,1.12$ & 87 & 79.1 & 23 & 20.9 & 1.03 & $.87,1.21$ \\
\hline$\$ 40000$ to $\$ 74999$ & 165 & 87.3 & 24 & 12.7 & 1.05 & $.93,1.19$ & 147 & 75.4 & 48 & 24.6 & .98 & $.84,1.14$ \\
\hline$\$ 75000$ or more & 279 & 89.1 & 34 & 10.9 & 1.07 & $.96,1.21$ & 244 & 77.2 & 72 & 22.8 & 1.00 & $.87,1.16$ \\
\hline \multicolumn{13}{|c|}{ Residence in Census Division } \\
\hline New England & 36 & 92.3 & 3 & 7.7 & 1.00 & & 33 & 80.5 & 8 & 19.5 & 1.00 & $\ldots$ \\
\hline Middle Atlantic & 89 & 85.6 & 15 & 14.4 & .93 & $.82,1.05$ & 86 & 79.6 & 22 & 20.4 & .99 & $.83,1.18$ \\
\hline East North Central & 89 & 84.0 & 17 & 16.0 & .91 & $.80,1.03$ & 83 & 77.6 & 24 & 22.4 & .96 & $.80,1.16$ \\
\hline West North Central & 29 & 74.4 & 10 & 25.6 & .81 & $.66, .99$ & 24 & 60.0 & 16 & 40.0 & .75 & $.56,1.00$ \\
\hline South Atlantic & 119 & 86.9 & 18 & 13.1 & .94 & $.84,1.05$ & 93 & 67.9 & 44 & 32.1 & .84 & $.70,1.02$ \\
\hline East South Central & 18 & 85.7 & 3 & 14.3 & .93 & $.76,1.13$ & 13 & 61.9 & 8 & 38.1 & .77 & $.53,1.11$ \\
\hline West South Central & 62 & 89.9 & 7 & 10.1 & .97 & $.86,1.10$ & 57 & 82.6 & 12 & 17.4 & 1.03 & $.85,1.24$ \\
\hline Mountain & 34 & 79.1 & 9 & 20.9 & .86 & $.72,1.02$ & 32 & 74.4 & 11 & 25.6 & .92 & $.73,1.17$ \\
\hline Pacific & 159 & 92.4 & 13 & 7.6 & 1.00 & $.91,1.11$ & 154 & 87.5 & 22 & 12.5 & 1.09 & $.93,1.28$ \\
\hline \multicolumn{13}{|l|}{ Residence in Southeast $^{c}$} \\
\hline No & 517 & 87.2 & 76 & 12.8 & 1.00 & & 484 & 80.0 & 121 & 20.0 & 1.00 & $\ldots$ \\
\hline Yes & 118 & 86.1 & 19 & 13.9 & 0.99 & $.92,1.06$ & 91 & 66.4 & 46 & 33.6 & .83 & $.73,0.94$ \\
\hline \multicolumn{13}{|c|}{ Exposure at Anatomical Site ${ }^{d}$} \\
\hline No & & & & & & & 15 & 68.2 & 7 & 31.8 & 1.00 & \\
\hline Yes & & & & & & & 560 & 77.8 & 160 & 22.2 & 1.14 & $.86,1.52$ \\
\hline \multicolumn{13}{|c|}{ Abbreviations: $\mathrm{Cl}$, confidence interval; PR, prevalence ratio; PrEP, pre-exposure prophylaxis; STI, sexually transmitted infection. } \\
\hline \multicolumn{13}{|c|}{$\begin{array}{l}\text { a Participants who provided blood samples for STI screening were considered to be possibly screened for syphilis, because blood samples are needed for syphilis screening, whereas swabs } \\
\text { and urine samples can be used for gold standard testing of gonorrhea and chlamydia. }\end{array}$} \\
\hline \multicolumn{13}{|c|}{${ }^{\mathrm{b}}$ Consistent screening defined as always or sometimes (versus rarely or never) being screened at PrEP care visits compared. } \\
\hline Alabama, Arkansas, Florida & 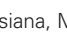 & 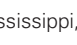 & & & & & & st Vir & & & & \\
\hline
\end{tabular}




\begin{tabular}{|c|c|c|c|c|c|c|c|c|c|c|c|c|}
\hline & \multicolumn{6}{|c|}{ Rectal STI Screening $(N=746)$} & \multicolumn{6}{|c|}{ Pharyngeal STI Screening ( $N=744)$} \\
\hline & \multicolumn{2}{|c|}{ Consistent } & \multicolumn{2}{|c|}{ Inconsistent } & \multirow[b]{2}{*}{ PR } & \multirow[b]{2}{*}{$95 \% \mathrm{Cl}$} & \multicolumn{2}{|c|}{ Consistent } & \multicolumn{2}{|c|}{ Inconsistent } & \multirow[b]{2}{*}{ PR } & \multirow[b]{2}{*}{$95 \% \mathrm{Cl}$} \\
\hline & N & $\%$ & N & $\%$ & & & N & $\%$ & N & $\%$ & & \\
\hline Total sample & 425 & 57.0 & 321 & 43.0 & & & 477 & 64.1 & 267 & 35.9 & & \\
\hline \multicolumn{13}{|l|}{ Age } \\
\hline $15-24$ & 61 & 67.0 & 30 & 33.0 & 1.00 & $\cdots$ & 63 & 70.0 & 27 & 30.0 & 1.00 & \\
\hline $25-34$ & 146 & 57.9 & 106 & 42.1 & .86 & $.72,1.03$ & 163 & 64.9 & 88 & 35.1 & .93 & $.79,1.09$ \\
\hline $35-44$ & 74 & 53.6 & 64 & 46.4 & .80 & $.65, .99$ & 89 & 64.5 & 49 & 35.5 & .92 & $.77,1.11$ \\
\hline $45-54$ & 91 & 55.2 & 74 & 44.8 & .82 & $.67,1.00$ & 102 & 61.8 & 63 & 38.2 & .88 & $.74,1.06$ \\
\hline $55-65$ & 53 & 53.0 & 47 & 47.0 & .79 & $.63,1.00$ & 60 & 60.0 & 40 & 40.0 & .86 & $.70,1.06$ \\
\hline \multicolumn{13}{|l|}{ Race/ethnicity } \\
\hline Black (Non-Hispanic) & 16 & 50.0 & 16 & 50.0 & 1.00 & $\cdots$ & 19 & 59.4 & 13 & 40.6 & 1.00 & \\
\hline Hispanic & 57 & 62.0 & 35 & 38.0 & 1.24 & $.85,1.82$ & 61 & 66.3 & 31 & 33.7 & 1.12 & $.81,1.54$ \\
\hline Other (Non-Hispanic) & 39 & 67.2 & 19 & 32.8 & 1.34 & $.91,1.99$ & 43 & 74.1 & 15 & 25.9 & 1.25 & $.90,1.73$ \\
\hline White (Non-Hispanic) & 313 & 55.5 & 251 & 44.5 & 1.11 & $.78,1.58$ & 354 & 63.0 & 208 & 37.0 & 1.06 & $.79,1.42$ \\
\hline \multicolumn{13}{|l|}{ Health insurance $^{a}$} \\
\hline None & 19 & 57.6 & 14 & 42.4 & 1.00 & $\ldots$ & 22 & 66.7 & 11 & 33.3 & 1.00 & $\ldots$ \\
\hline Public & 58 & 50.0 & 58 & 50.0 & .87 & $.62,1.23$ & 74 & 63.8 & 42 & 36.2 & .96 & $.73,1.26$ \\
\hline Private & 347 & 58.4 & 247 & 41.6 & 1.01 & $.75,1.37$ & 380 & 64.2 & 212 & 35.8 & .96 & $.75,1.23$ \\
\hline \multicolumn{13}{|c|}{ Annual household Income } \\
\hline$\$ 0$ to $\$ 19999$ & 35 & 53.0 & 31 & 47.0 & 1.00 & $\ldots$ & 44 & 66.7 & 22 & 33.3 & 1.00 & $\ldots$ \\
\hline$\$ 20000$ to $\$ 39999$ & 59 & 53.6 & 51 & 46.4 & 1.01 & $.76,1.35$ & 62 & 56.4 & 48 & 43.6 & .85 & $.67,1.07$ \\
\hline$\$ 40000$ to $\$ 74999$ & 109 & 55.9 & 86 & 44.1 & 1.05 & $.81,1.37$ & 125 & 64.4 & 69 & 35.6 & .97 & $.79,1.18$ \\
\hline$\$ 75000$ or more & 187 & 58.8 & 131 & 41.2 & 1.11 & $.87,1.42$ & 206 & 65.0 & 111 & 35.0 & .97 & $.81,1.18$ \\
\hline \multicolumn{13}{|c|}{ Residence in census Division } \\
\hline New England & 29 & 70.7 & 12 & 29.3 & 1.00 & & 30 & 73.2 & 11 & 26.8 & 1.00 & \\
\hline Middle Atlantic & 65 & 60.2 & 43 & 39.8 & .85 & $.66,1.09$ & 74 & 69.2 & 33 & 30.8 & .95 & $.76,1.18$ \\
\hline East North Central & 57 & 52.8 & 51 & 47.2 & .75 & $.57,0.97$ & 59 & 54.6 & 49 & 45.4 & .75 & $.58, .96$ \\
\hline West North Central & 21 & 52.5 & 19 & 47.5 & .74 & $.52,1.06$ & 24 & 61.5 & 15 & 38.5 & .84 & $.62,1.15$ \\
\hline South Atlantic & 65 & 46.1 & 76 & 53.9 & .65 & $.50, .85$ & 79 & 56.0 & 62 & 44.0 & .77 & $.60, .97$ \\
\hline East South Central & 7 & 33.3 & 14 & 66.7 & .47 & $.25, .89$ & 9 & 42.9 & 12 & 57.1 & .59 & $.35, .99$ \\
\hline West South Central & 40 & 58.0 & 29 & 42.0 & .82 & $.62,1.09$ & 47 & 68.1 & 22 & 31.9 & .93 & $.73,1.19$ \\
\hline Mountain & 19 & 45.2 & 23 & 54.8 & .64 & $.43, .94$ & 20 & 47.6 & 22 & 52.4 & .65 & $.45, .94$ \\
\hline Pacific & 122 & 69.3 & 54 & 30.7 & .98 & $.79,1.22$ & 135 & 76.7 & 41 & 23.3 & 1.05 & $.86,1.28$ \\
\hline \multicolumn{13}{|l|}{ Residence in Southeast ${ }^{\mathrm{b}}$} \\
\hline No & 364 & 60.1 & 242 & 39.9 & 1.00 & $\cdots$ & 400 & 66.2 & 204 & 33.8 & 1.00 & \\
\hline Yes & 61 & 43.6 & 79 & 56.4 & .73 & $.59, .89$ & 77 & 55.0 & 63 & 45.0 & .83 & $.71, .97$ \\
\hline \multicolumn{13}{|c|}{ Exposure at Anatomical Site ${ }^{c}$} \\
\hline No & 61 & 44.2 & 77 & 55.8 & 1.00 & $\ldots$ & 15 & 62.5 & 9 & 37.5 & 1.00 & $\ldots$ \\
\hline Yes & 364 & 59.9 & 244 & 40.1 & 1.35 & $1.11,1.65$ & 462 & 64.2 & 258 & 35.8 & 1.03 & $.75,1.41$ \\
\hline \multirow{2}{*}{\multicolumn{13}{|c|}{$\begin{array}{l}\text { Abbreviations: } \mathrm{Cl} \text {, confidence interval; PR, prevalence ratio; PrEP, pre-exposure prophylaxis; } \mathrm{STI} \text {, sexually transmitted infection. } \\
{ }^{a} \text { Consistent screening defined as always or sometimes (vs rarely or never) being screened at PrEP care visits compared. }\end{array}$}} \\
\hline & & & & & & & & & & & & \\
\hline $\begin{array}{l}{ }^{b} \text { Alabama, Arkansas, Florida } \\
{ }^{\circ} \text { Defined as any receptive ar }\end{array}$ & $c_{k} y_{1}$ & ana, I & 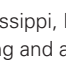 & 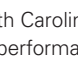 & 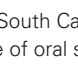 & & & . & & & & \\
\hline
\end{tabular}

MSM reporting consistent screening of pharyngeal STIs were about the same between MSM reporting sexual exposure and none at the pharynx (64\% vs 63\%).

Among MSM who had ever used PrEP, age, race/ethnicity, health insurance, annual household income, census division, and residence in Southeast states were not significantly associated with possible syphilis screening at PrEP care visits (Table 3). For urogenital, rectal, and pharyngeal STIs, there was a general trend of MSM aged 15-24 years reporting consistent screening compared to MSM aged 55-65 years at PrEP care visits (Tables 3 and 4). In unadjusted analyses, MSM in Southeast states reported a lower prevalence of consistent urogenital (prevalence ratio [PR], 0.83; 95\% confidence interval [CI], .73-.94), rectal (PR, 0.73; 95\% CI, .59-.89), and pharyngeal (PR, 0.83; 95\% CI, .71-.97) STI screening during PrEP care (Table 4). MSM who reported exposure at the rectum reported a 35\% higher prevalence of consistent rectal STI screening at PrEP care visits (PR, 1.35; 95\% CI, 1.11-1.65). Prevalence of 
consistent STI screening was not substantially different between MSM reporting exposure at anatomical site compared to those with no exposure for urogenital (PR, 1.14; 95\% CI, .86-1.52) and pharyngeal STIs (PR, 1.03; 95\% CI, .75-1.41) (Tables 3 and 4 ).

In adjusted models, a smaller proportion of MSM in Southeast states reported consistent STI screening compared to MSM in other regions (Table 5). MSM in the Southeast had 14\% lower prevalence of consistent urogenital STI screening (adjusted prevalence ratio [aPR], 0.86; 95\% CI, .76-.98) and 24\% lower prevalence of consistent rectal STI screening (aPR, 0.76; 95\% CI, .62-.93) during PrEP care. A lower prevalence of MSM in Southeast states compared to all other regions of the country reported consistent pharyngeal STI screening during PrEP care (aPR, 0.87; 95\% CI, .74-1.03). The prevalence of consistent possible syphilis screening was not substantially different between MSM in the Southeast and MSM in all other regions (aPR, 1.02; 95\% CI, .95-1.10).

\section{DISCUSSION}

In this national study of MSM in the United States, consistency of STI screening at PrEP care visits was lower than recommended, especially for rectal and pharyngeal infections that are mostly asymptomatic. Our findings also highlight the regional variation in gaps between recommendations and PrEP clinical

\section{Table 5. Multivariable Associations of Consistent ${ }^{\mathrm{a}}$ Sexually Transmitted Infection Screening With Residence in the Southeast United States ${ }^{b}$ Among Men Who Have Sex With Men Who Have Ever Used Pre-exposure Prophylaxis}

\begin{tabular}{|c|c|c|}
\hline & Adjusted PR ${ }^{c}$ & $95 \% \mathrm{Cl}$ \\
\hline \multicolumn{3}{|l|}{ Possible syphilis screening ${ }^{d, e}$} \\
\hline Residence outside Southeast & 1.00 & $\ldots$ \\
\hline Residence in Southeast & 1.02 & $.95,1.10$ \\
\hline \multicolumn{3}{|l|}{ Urogenital STI screening } \\
\hline Residence outside Southeast & 1.00 & $\ldots$ \\
\hline Residence in Southeast & .86 & $.76, .98$ \\
\hline \multicolumn{3}{|l|}{ Rectal STI screening } \\
\hline Residence outside Southeast & 1.00 & $\cdots$ \\
\hline Residence in Southeast & .76 & $.62, .93$ \\
\hline \multicolumn{3}{|l|}{ Pharyngeal STI screening } \\
\hline Residence outside Southeast & 1.00 & $\ldots$ \\
\hline Residence in Southeast & .87 & $.74,1.03$ \\
\hline \multicolumn{3}{|c|}{$\begin{array}{l}\text { Abbreviations: } \mathrm{Cl} \text {, confidence interval; PR, prevalence ratio; PrEP, pre-exposure prophy- } \\
\text { laxis; STI, sexually transmitted infection. }\end{array}$} \\
\hline \multicolumn{3}{|c|}{$\begin{array}{l}{ }^{a} \text { Consistent screening defined as always or sometimes (vs rarely or never) being screened } \\
\text { at PrEP care visits compared. }\end{array}$} \\
\hline \multicolumn{3}{|c|}{$\begin{array}{l}\text { besidence in Alabama, Arkansas, Florida, Georgia, Kentucky, Louisiana, Mississippi, } \\
\text { North Carolina, South Carolina, Tennessee, Virginia, and West Virginia. }\end{array}$} \\
\hline \multirow{2}{*}{\multicolumn{3}{|c|}{$\begin{array}{l}{ }^{c} \text { Adjusted for age, race/ethnicity, annual household income, and sexual exposure at an- } \\
\text { atomical site. } \\
{ }^{d} \text { Not adjusted for sexual exposure at anatomical site. }\end{array}$}} \\
\hline${ }^{\mathrm{d}}$ Not adjusted for sexual exposure at a & & \\
\hline \multicolumn{3}{|c|}{$\begin{array}{l}\text { e Participants who provided blood samples for STI screening were considered to be pos- } \\
\text { sibly screened for syphilis, because blood samples are needed for syphilis screening, } \\
\text { whereas swabs and urine samples can be used for gold standard testing of gonorrhea } \\
\text { and chlamydia. }\end{array}$} \\
\hline
\end{tabular}

practice overall and raise concerns about whether comprehensive PrEP care as currently practiced would be effective for STI control, particularly in the Southeast where the burden of bacterial STIs is highest $[1,18]$.

This study contributes to the growing literature on patterns of STI screening within PrEP care. With the exception of one national study [14], research has been limited to individual clinics or health systems, often with limited sample sizes $[7,13,15,16]$. These studies analyze a variety of data streams, including electronic health records, health insurance claims, or laboratory screening $[15,16,27]$. Many different measures have been used as primary outcomes: screening at last PrEP care visit [13], STI or rectal STI screening in the prior 12 months [7], or proportion screened for STIs within 6 months of PrEP initiation [14]. Our study, in contrast, measured STI screening across PrEP care visits that may better reflect ongoing consistency of clinical practice. We also stratified data by current and prior PrEP use, and more frequent STI screening among current PrEP users compared to prior users was expected given CDC's revised recommendations to more frequent STI screening in 2017.

Despite these different study designs and outcomes, research has consistently shown suboptimal STI screening among PrEP users, especially for extragenital (rectal or pharyngeal) screening [13-16]. Similar to a study conducted in Baltimore, we found that among all site-specific screening, rectal swabs were reported least often [16]. A study in New York City, in contrast, found that pharyngeal STI screening was the least reported (at the last PrEP care visit) [13]. These results are also consistent with findings of infrequent extragenital STI screening among MSM outside of PrEP care [28-30]. Infrequent extragenital screening is a public health concern because of the higher prevalence of asymptomatic chlamydia or gonorrhea infections that may remain undetected [31]. Asymptomatic STIs at these sites also lead to increased risk of HIV infection among partners in the sexual network not using PrEP [24, 32].

Several factors have been found to be associated with more recent or comprehensive STI screening: younger age, white race, college education, reporting exposure at anatomical sites that may trigger screening, and previous syphilis diagnoses $[7,13,15,16]$. We additionally found that residence in the Southeast was associated with inconsistent urogenital and rectal STI screening, after adjusting for potential confounders. These results have implications for regional improvements of comprehensive PrEP care at the patient, provider, and systems level. MSM have previously noted discomfort in discussing their sexual orientation and subsequently sexual health and behaviors with their primary care providers, emphasizing the importance of patient-provider communication in PrEP care [33]. At the provider level, time constraints, cultural and language barriers, difficulty obtaining a sexual history, and patient privacy concerns have affected HIV providers' ability to conduct routine STI screening [34]. The cost of ancillary 
services associated with PrEP care such as STI screening may also be a barrier, as providers have noted that high cost of medication and health insurance coverage may hinder the real-world effectiveness of PrEP [35]. Related to cost, alternative options should also be considered for undocumented populations. Moreover, 7 of the 12 Southeast states (in our definition) have not expanded Medicaid under the Affordable Care Act, potentially limiting the ability of MSM to obtain adequate coverage for consistent STI screening [36]. Gaps in comprehensive PrEP care may exacerbate the large racial and socioeconomic disparities in HIV and STI incidence in the region [37]. Wider implementation of alternative STI screening approaches, such as self-collected rectal and pharyngeal swabs or home-screening kits, may also improve STI screening coverage for PrEP-using MSM [38, 39].

\section{Limitations}

This study has several limitations. First, this web-based sample does not necessarily represent the larger population of MSM with respect to age, race/ethnicity, health insurance, annual household income, and geographic residential location. Most participants were white, had higher income levels, had private health insurance, and lived in the mid-Atlantic/Northeast or the West Coast. Compared to venue-based sampling, web-based sampling of MSM may yield a sample with more non-Hispanic white and higher-income men [40]. The survey was Englishonly, which may have contributed to the lack of ethnic representativeness of the responses. Additionally, given our focus on the Southeast and that $4 \%$ of respondents are Black, our sample critically underrepresents the MSM population in the Southeast. Second, stratifying participant data by the covariates of interest yielded small sample sizes in some categories (reflected in wide confidence intervals for some estimates). Third, we relied on self-reported data, and there may be misclassification of screening frequency due to not understanding what specific STIs are being screened. We may be overestimating consistency of possible syphilis screening, because participants may misinterpret blood draws for HIV screening or other tests at PrEP care visits as inclusive of STI screening. Fourth, the data were also limited in classifying anatomical site exposure because the ARTnet study collected data on a maximum of 5 partners; this may not align with their PrEP care visits. Fifth, we assumed that PrEP users accessed PrEP in their reported residential ZIP Code, but it is possible that prior or current PrEP users accessed PrEP in a different state. Sixth, respondents may have received STI screening outside of PrEP care (eg, through another healthcare provider or community-based organization), the frequency of which is not captured in ARTnet. Finally, the STI screening frequency measure (always, sometimes, rarely, or never) allows for an approximate but not perfect estimate of adherence to CDC recommendations, particularly because "sometimes" can represent a wide range of screening frequencies as interpreted by respondents. For this reason, we used the term "consistent" rather than "as recommended."

\section{CONCLUSIONS}

Data from this large national study of MSM improved the understanding of geographic differences in comprehensive health services for MSM using PrEP. Although a higher proportion of PrEP users reported recent STI screening compared to non-PrEP users, barriers exist to complete adherence to CDC PrEP guidelines of comprehensive screening for STIs at all exposure sites among MSM. This was especially true in Southeast states where the STI burden is high and where the federal government has prioritized funding HIV prevention programs through the Ending the HIV Epidemic initiative [21]. As PrEP is scaled up in the Southeast through this national initiative, resources at the patient, provider, and systems level to support STI screening and treatment as part of PrEP care should simultaneously be strengthened. Otherwise, suboptimal STI screening in PrEP care may lead to increases in overall STI incidence among MSM.

\section{Notes}

Financial support. This work was supported by the National Institutes of Health (grant number R21 MH112449).

Potential conflicts of interest. C. K. reports grants from Gilead Sciences, $\mathrm{NIH}$, and CDC, outside the submitted work. J. M. reports consulting for Kaiser Permanente Northern California on a research grant from Gilead Sciences, outside the submitted work. All other authors report no conflicts of interest. All authors have submitted the ICMJE Form for Disclosure of Potential Conflicts of Interest. Conflicts that the editors consider relevant to the content of the manuscript have been disclosed.

\section{References}

1. Centers for Disease Control and Prevention. Sexually Transmitted Disease Surveillance 2018. US Dep Health Hum Serv 2019. Available at: https://www.cdc. gov/std/stats. Accessed 4 March 2020.

2. Centers for Disease Control and Prevention. HIV Surveillance Report, 2018 (Preliminary). Centers for Disease Control and Prevention, 2019. Available at: http://www.cdc.gov/hiv/library/reports/hiv-surveillance.html. Accessed 4 March 2020 .

3. Purcell DW, Johnson CH, Lansky A, et al. Estimating the population size of men who have sex with men in the United States to obtain HIV and syphilis rates. Open AIDS J 2012; 6:98-107.

4. Ong JJ, Baggaley RC, Wi TE, et al. Global epidemiologic characteristics of sexually transmitted infections among individuals using preexposure prophylaxis for the prevention of HIV infection: a systematic review and meta-analysis. JAMA Netw Open 2019; 2:e1917134.

5. Montaño MA, Dombrowski JC, Dasgupta S, et al. Changes in sexual behavior and STI diagnoses among MSM initiating PrEP in a clinic setting. AIDS Behav 2019; 23:548-55.

6. Marcus JL, Hurley LB, Hare CB, et al. Preexposure prophylaxis for HIV prevention in a large integrated health care system: adherence, renal safety, and discontinuation. J Acquir Immune Defic Syndr 1999 2016; 73:540-6.

7. Menza TW, Lipira L, Bhattarai A, Ramirez FJ, Orellana ER. Self-reported screening for rectal sexually transmitted infections among men who have sex with men. Sex Transm Dis 2019; 46:683-8.

8. Volk JE, Marcus JL, Phengrasamy T, et al. No new HIV infections with increasing use of HIV preexposure prophylaxis in a clinical practice setting. Clin Infect Dis 2015; 61:1601-3.

9. Paz-Bailey G, Mendoza MC, Finlayson T, et al; NHBS Study Group. Trends in condom use among MSM in the United States: the role of antiretroviral therapy and seroadaptive strategies. AIDS 2016; 30:1985-90. 
10. Harawa NT, Holloway IW, Leibowitz A, et al. Serious concerns regarding a meta-analysis of preexposure prophylaxis use and STI acquisition. AIDS 2017; 31:739-40.

11. Centers for Disease Control and Prevention. Preexposure prophylaxis for the prevention of HIV infection in the United States-2017 update: a clinical practice guideline. 2018. Available at: https://www.cdc.gov/hiv/pdf/risk/prep/cdc-hivprep-guidelines-2017.pdf. Accessed 4 March 2020.

12. Jenness SM, Weiss KM, Goodreau SM, et al. Incidence of gonorrhea and chlamydia following human immunodeficiency virus preexposure prophylaxis among men who have sex with men: a modeling study. Clin Infect Dis 2017; 65:712-8.

13. Parsons JT, John SA, Whitfield THF, Cienfuegos-Szalay J, Grov C. Human immunodeficiency virus/sexually transmitted infection counseling and testing services received by gay and bisexual men using preexposure prophylaxis at their last PrEP care visit. Sex Transm Dis 2018; 45:798-802.

14. Huang YA, Tao G, Samandari T, Hoover KW. Laboratory testing of a cohort of commercially insured users of HIV preexposure prophylaxis in the United States, 2011-2015. J Infect Dis 2018; 217:617-21.

15. Spinelli MA, Scott HM, Vittinghoff E, et al. Provider adherence to pre-exposure prophylaxis monitoring guidelines in a large primary care network. Open Forum Infect Dis 2018; 5: ofy099.

16. Schumacher C, Wu L, Chandran A, et al. STI screening among gay, bisexual and other men who have sex with men prescribed PrEP in Baltimore City, Maryland. Clin Infect Dis 2020; 71:2637-44.

17. Sullivan PS, Mena L, Elopre L, Siegler AJ. Implementation strategies to increase PrEP uptake in the South. Curr HIV/AIDS Rep 2019; 16:259-69.

18. Marotta P. Assessing spatial relationships between race, inequality, crime, and gonorrhea and chlamydia in the United States. J Urban Health 2017; 94:683-98.

19. Patel RR, Mena L, Nunn A, et al. Impact of insurance coverage on utilization of pre-exposure prophylaxis for HIV prevention. PLoS One 2017; 12:e178737.

20. Philbin MM, Parker CM, Parker RG, Wilson PA, Garcia J, Hirsch JS. The Promise of pre-exposure prophylaxis for black men who have sex with men: an ecological approach to attitudes, beliefs, and barriers. AIDS Patient Care STDS 2016; 30:282-90.

21. Fauci AS, Redfield RR, Sigounas G, Weahkee MD, Giroir BP. Ending the HIV epidemic: a plan for the United States. JAMA 2019; 321:844-5.

22. Weiss KM, Goodreau SM, Morris M, et al. Egocentric sexual networks of men who have sex with men in the United States: results from the ARTnet study Epidemics 2020; 30:100386.

23. Zlotorzynska M, Sullivan P, Sanchez T. The annual American Men's internet survey of behaviors of men who have sex with men in the United States: 2016 key indicators report. JMIR Public Health Surveill 2019; 5:e11313.

24. Jenness SM, Weiss KM, Prasad P, Zlotorzynska M, Sanchez T. Bacterial sexually transmitted infection screening rates by symptomatic status among men who have sex with men in the United States: a hierarchical Bayesian analysis. Sex Transm Dis 2019; 46:25-30.

25. BEA Regions. Available at: https://apps.bea.gov/regional/docs/regions.cfm Accessed 4 March 2020
26. R Core Team. R: a language and environment for statistical computing. Vienna Austria: R Foundation for Statistical Computing, 2019. Available at: https:// www.R-project.org/. Accessed 4 March 2020.

27. Hevey MA, Walsh JL, Petroll AE. PrEP continuation, HIV and STI testing rates, and delivery of preventive care in a clinic-based cohort. AIDS Educ Prev 2018; 30:393-405.

28. de Voux A, Bernstein KT, Kirkcaldy RD, Zlotorzynska M, Sanchez T. Self-reported extragenital chlamydia and gonorrhea testing in the past 12 months among men who have sex with men in the United States: American Men's internet survey, 2017. Sex Transm Dis 2019; 46:563-70.

29. Barbee LA, Dhanireddy S, Tat SA, Marrazzo JM. Barriers to bacterial sexually transmitted infection testing of HIV-infected men who have sex with men engaged in HIV primary care. Sex Transm Dis 2015; 42:590-4.

30. Patton ME, Kidd S, Llata E, et al. Extragenital gonorrhea and chlamydia testing and infection among men who have sex with men-STD Surveillance Network, United States, 2010-2012. Clin Infect Dis 2014; 58:1564-70.

31. Kent CK, Chaw JK, Wong W, et al. Prevalence of rectal, urethral, and pharyngeal chlamydia and gonorrhea detected in 2 clinical settings among men who have sex with men: San Francisco, California, 2003. Clin Infect Dis 2005; 41:67-74.

32. Bernstein KT, Marcus JL, Nieri G, Philip SS, Klausner JD. Rectal gonorrhea and chlamydia reinfection is associated with increased risk of HIV seroconversion. J Acquir Immune Defic Syndr 1999 2010; 53:537-43.

33. Maloney KM, Krakower DS, Ziobro D, Rosenberger JG, Novak D, Mayer KH Culturally competent sexual healthcare as a prerequisite for obtaining preexposure prophylaxis: findings from a qualitative study. LGBT Health 2017; 4:310-4.

34. Carter JW Jr, Hart-Cooper GD, Butler MO, Workowski KA, Hoover KW. Provider barriers prevent recommended sexually transmitted disease screening of HIVinfected men who have sex with men. Sex Transm Dis 2014; 41:137-42.

35. Krakower D, Ware N, Mitty JA, Maloney K, Mayer KH. HIV providers' perceived barriers and facilitators to implementing pre-exposure prophylaxis in care settings: a qualitative study. AIDS Behav 2014; 18:1712-21.

36. Kaiser Family Foundation. Status of state Medicaid expansion decisions: interactive map. Henry J Kais Fam Found 2020. Available at: https://www.kff.org/medicaid/issue-brief/status-of-state-medicaid-expansion-decisions-interactive-map/. Accessed 4 March 2020

37. Goodreau SM, Rosenberg ES, Jenness SM, et al. Sources of racial disparities in HIV prevalence in men who have sex with men in Atlanta, GA, USA: a modelling study. Lancet HIV 2017; 4:e311-20.

38. Moncada J, Schachter J, Liska S, Shayevich C, Klausner JD. Evaluation of self-collected glans and rectal swabs from men who have sex with men for detection of chlamydia trachomatis and Neisseria gonorrhoeae by use of nucleic acid amplification tests. J Clin Microbiol 2009; 47:1657-62.

39. Fisher M, Wayal S, Smith H, et al; Brighton Home Sampling Kit Project Steering Group. Home sampling for sexually transmitted infections and HIV in men who have sex with men: a prospective observational study. PLoS One 2015; 10:e0120810.

40. Chen YT, Bowles K, An Q, et al. Surveillance among men who have sex with men in the United States: a comparison of web-based and venue-based samples. AIDS Behav 2018; 22:2104-12. 